(8) Flora. Nov, 26, $8^{\mathrm{h}} 40^{\mathrm{m}}$. Durch Wind das Fernrohr zeitweise bewegt, daher Messung unsicher.

(38) Leda. Febr. 24. Anschluss in AR. und Decl. durch ${ }_{4}$ Doppeleinstellungen mit der Mikrometerschraube.

(106) Dione. Sept. 3. Anschluss in AR. und Decl. durch 9 Doppeleinstellungen mit der Mikrometerschraube.

München 1897 Juni 6.
(385) Ilmatar. Aug. $28,12^{\mathrm{b}} 43^{\mathrm{m}}$. Anschluss in AR. und Decl. durch 7 Doppeleinstellungen mit der Mikrometerschraube.

Die Ephemeriden von Prof. Neugebauer sind mir von Prof. R. Luther guitigst mitgetheilt worden.

\title{
Sternbedeckungen durch den Mond
}

beobachtet am Utrechter Refractor von Dr. A. A. Nyland.

(Mitgetheilt von Prof. F.A.C. Oudemans.)

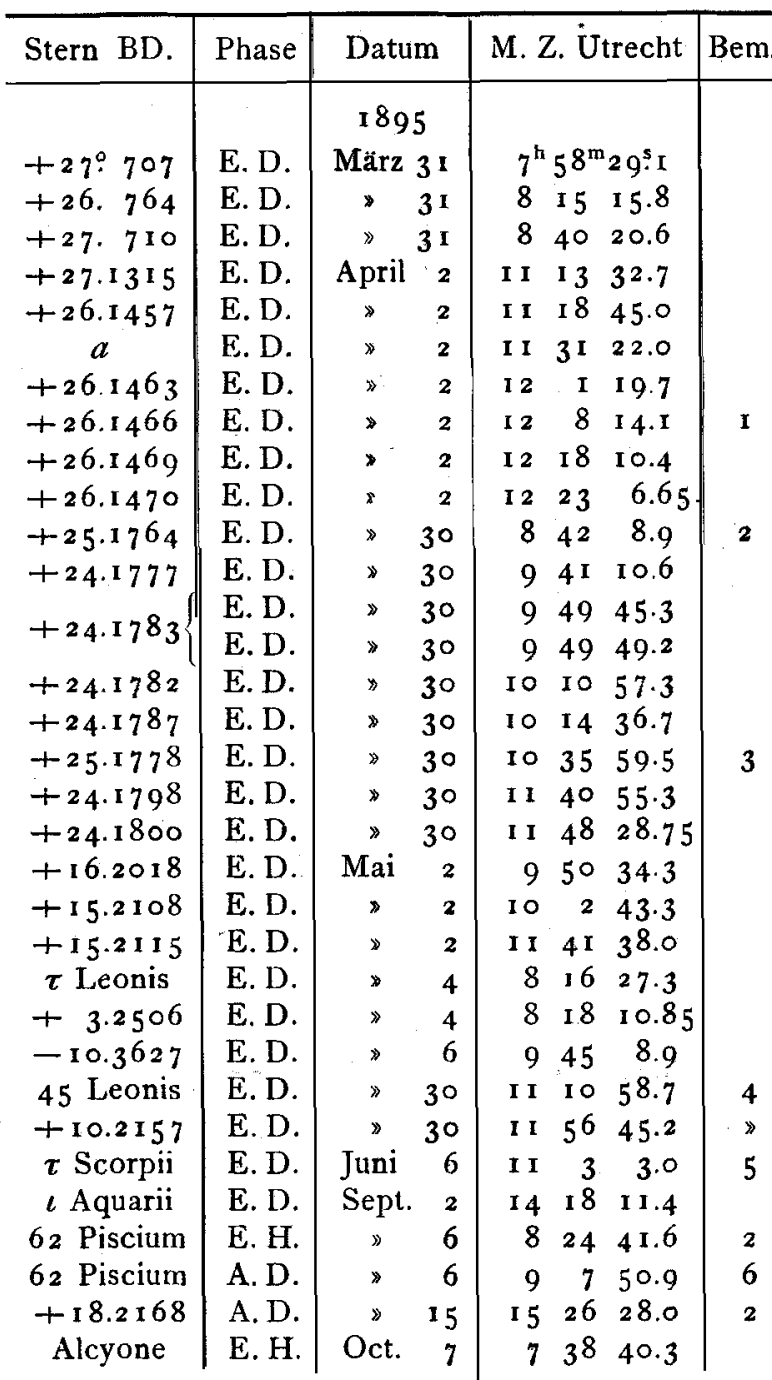

\begin{tabular}{|c|c|c|c|c|c|c|c|}
\hline Stern BD. & Phase & \multicolumn{2}{|c|}{ Datum } & \multicolumn{3}{|c|}{ M. Z. Utrecht } & Bem \\
\hline & & \multicolumn{2}{|c|}{1895} & \multirow{2}{*}{\multicolumn{3}{|c|}{$7^{\mathrm{h}} 42^{\mathrm{m}} \quad 3^{\mathrm{s}} \cdot 7$}} & \\
\hline 4 Plejadum & A. D. & Oct. & 7 & & & & 2,4 \\
\hline c Plejadum & A. D. & 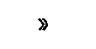 & 7 & 7 & 46 & 39.9 & \\
\hline $3^{2}$ Plejadum & A. D. & $\gg$ & 7 & 9 & 17 & 31.7 & 4 \\
\hline - I $4: 6$ I 78 & E. D. & Nov. & 23 & 7 & 6 & 9.0 & 7 \\
\hline-15.6110 & E. D. & $\gg$ & 23 & 7 & 34 & 13.0 & 8 \\
\hline-10.5966 & E. D. & $\gg$ & 24 & 5 & 17 & 8.2 & \\
\hline I Piscium & E. D. & 189 & 25 & 10 & I I & 35.2 & 9 \\
\hline+330 & E. D. & Jan. & 20 & 7 & 47 & I $3 \cdot 3$ & \\
\hline+3.33 & E. D. & 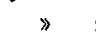 & 20 & 8 & 16 & 39.2 & 2 \\
\hline $\begin{array}{r}34 \\
+\quad 3.34\end{array}$ & E. D. & " & 20 & 8 & 35 & I I . 4 & 2 \\
\hline & E. D. & $»$ & 23 & 9 & 44 & $1 \cdot 3$ & ro \\
\hline+19.391 & E. D. & $\gg$ & 23 & 9 & 57 & 26.6 & \\
\hline+19.388 & E. D. & " & 23 & 10 & I & 42.5 & 2 \\
\hline+19.392 & E. D. & » & 23 & Io & 22 & $49 \cdot I$ & I I \\
\hline+19.393 & E. D. & 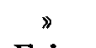 & 23 & Io & 24 & 22.9 & I I \\
\hline+25.674 & E. D. & Febr. & $2 \mathrm{I}$ & 10 & 45 & 22.4 & I I \\
\hline $\begin{array}{r}-27.1154 \\
\end{array}$ & E. D. & März & 22 & 7 & 2 I & $9 \cdot 4$ & I 2 \\
\hline$c$ & E. D. & $\gg$ & 22 & 7 & 29 & 13.8 & II \\
\hline+27.1158 & E. D. & $\triangleright$ & 22 & 7 & $3^{8}$ & $0 . \mathbf{I}$ & \\
\hline$d$ & E. D. & 2 & 22 & 7 & 49 & $31 \cdot 3$ & I 3 \\
\hline & E. D. & * & 22 & 7 & 50 & 57.7 & 13 \\
\hline+27.1101 & E. D. & 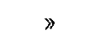 & 22 & 7 & 51 & $43^{2}$ & I 3 \\
\hline+27.1164 & E. D. & $\nabla$ & 22 & 8 & 9 & I 8.8 & 8 \\
\hline$e$ & E. D. & 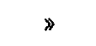 & 22 & 8 & 10 & $27 \cdot 3$ & 2 \\
\hline+25.1708 & E. D. & $»$ & 23 & 7 & 23 & 45 & 8 \\
\hline+25.1709 & E. D. & $>$ & 23 & 7 & 26 & 22.7 & 1 I \\
\hline+25.1713 & E. D. & * & 23 & 8 & 4 & r 4.3 & I I \\
\hline+24.1707 & E. D. & $»$ & 23 & 8 & 33 & 19.7 & 14 \\
\hline 79 Leonis & E. D. & I 89 & $\begin{array}{l}27 \\
7\end{array}$ & 10 & 20 & 43.6 & \\
\hline+22.1771 & $\begin{array}{l}\text { E. D. } \\
\text { E. D. }\end{array}$ & Mai & 7 & 8 & 40 & 55.4 & 13 \\
\hline & & & 7 & 9 & & $3^{6.6}$ & \\
\hline
\end{tabular}

Bemerkungen.

I. Der Stern ist nach BD. $9 \mathrm{~m}_{2}$; ich habe ihn an voll; niedrig. Der Rand wallte stark. Eintritt sebr unsicher. 8 Abenden (April 1895 ) immer $=$ BD. +26.1466 und -6 . Vielleicht ist die Zeitangabe um ro Schläge des 1469, also etwa 8.85 geschätzt. - 2. Unsicher. - 3 . Der Stern verschwand in der Nähe des nördlichen Horns, und ist ungefähr $10^{\mathrm{h}} 40^{\mathrm{m}}$ noch am dunklen Rande wieder sichtbar geworden. - 4. Wolken. - 5. Mond beinahe Chronometers $\left(=4^{5} \cdot 6\right.$ ) zu verbessern, und muss also $46^{5} \cdot 3$ anstatt 50.5 gelesen werden. - 7. Ziemlich unsicher. Stern sehr schwach. Der Mondrand wallte stark. - 8. Der Stern verschwand allmählig, kam noch einmal zum Vorschein und 
verschwand dann plötzlich. Die angegebene Zeit bezieht sich auf dieses letzte Verschwinden. - 9. Luft sehr unrubig. Der Stern verschwand langsam; die erste Lichtabnahme trat schon $10^{\mathrm{h}}{ }_{1} \mathrm{I}^{\mathrm{m}} 34^{\mathrm{s}}$ ein. - ro. Scharf. - I1. Sehr scharf. 12. Stern verschwand allmählig. - 13. Nicht ganz scharf. - 14 . Stern verschwand ziemlich langsam.

$\mathrm{E}=$ Eintritt, $\mathrm{A}=$ Austritt.

$\mathrm{D}=$ am dunklen Rande, $\mathrm{H}=$ am hellen Rande.

Die Zahlen der vierten Spalte sind in mittlerer Ut. rechter Zeit gegeben. Die Länge von Utrecht östlich von Greenwich ist $0^{\mathrm{h}} 2 \mathrm{O}^{\mathrm{m}} 3{ }^{\mathrm{s}} \cdot 7$, die Breite $+52^{\circ} 5^{\prime} 9.5$. Die

Utrecht 1897 Juli 22.
Sterne zehnter Grösse $a, b, c, d$ und $e$ kommen nicht in der Bonner Durchmusterung vor. Für 1855.0 sind ihre Positionen beiläufig:

\begin{tabular}{|c|c|c|}
\hline Stern & $\alpha$ & $\delta$ \\
\hline$a$ & $6^{\mathrm{h}} 5^{8^{\mathrm{m}} 25^{\mathrm{s}}}$ & $+27^{\circ} 9 \cdot 5$ \\
\hline$b$ & 229 I 2 & + I93 3.3 \\
\hline$c$ & $\begin{array}{lll}6 & 25 & 47\end{array}$ & +276.3 \\
\hline$d$ & $\begin{array}{lll}6 & 26 & 5\end{array}$ & $+27 \times 3.5$ \\
\hline$e$ & $\begin{array}{lll}6 & 26 & 53\end{array}$ & +2729.7 \\
\hline
\end{tabular}

A. A. Nyland.

Cordoba Observations of Comet $1897 \mathrm{I}$.

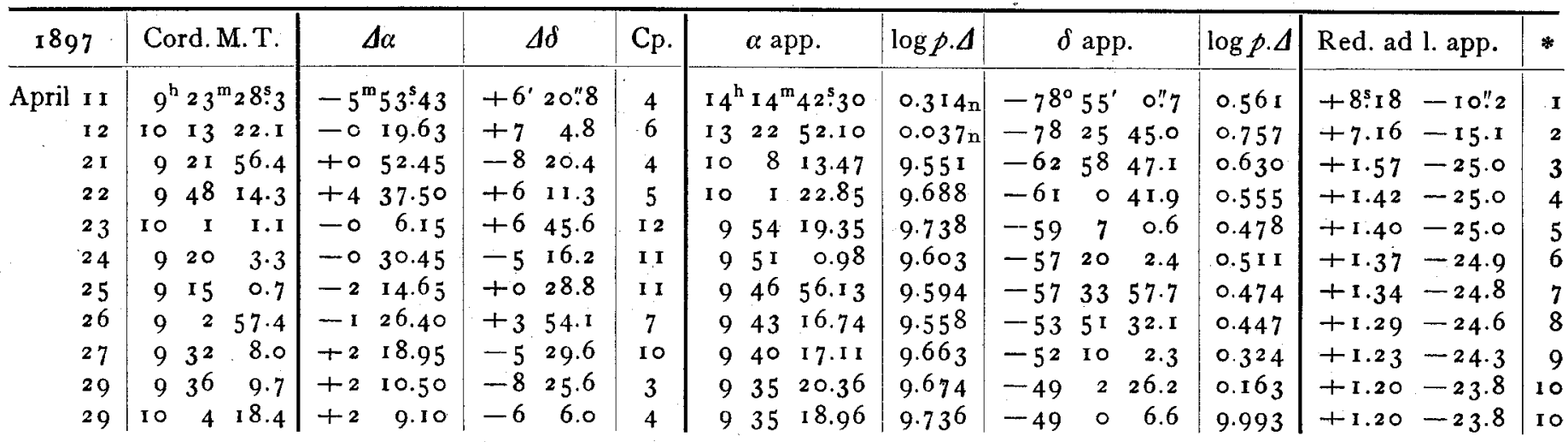

Mean places of the comparison stars.

\begin{tabular}{|c|c|c|c|}
\hline * & $\alpha 1897.0$ & $\delta 1897.0$ & Authority \\
\hline I & $14^{h} 20^{m} 27: 55$ & $-79^{\circ} \quad I^{\prime}$ x I I" 3 & Cord.ZC. I I 4 I (I), MC. (4) \\
\hline 2 & I 3234.57 & $\begin{array}{lll}-78 & 32 & 34.7\end{array}$ & Cord. GC. 18322 \\
\hline 3 & 10 $7 \quad 19.54$ & $\begin{array}{lll}-62 & 50 & 1.7\end{array}$ & Cord. ZC. 497 \\
\hline 4 & $\begin{array}{lll}9 & 56 & 43.93\end{array}$ & $\begin{array}{lll}-61 & 6.28 .2\end{array}$ & Cord. GC. 13673 \\
\hline 5 & 95424.10 & $-59 \quad 1321.2$ & Cord. ZC. 4 I 35 \\
\hline
\end{tabular}

\begin{tabular}{|c|c|c|c|}
\hline$*$ & $\alpha 1897.0$ & $\delta 1897.0$ & Authority \\
\hline 6 & $9^{\mathrm{b}} 5^{\mathrm{I}^{\mathrm{m}}} 30.06$ & $-57^{\circ} 14^{\prime} 21 "$ I & Cord. ZC. 3920 \\
\hline 7 & $\begin{array}{lll}9 & 49 & 9.44\end{array}$ & $\begin{array}{lll}-55 & 34 & \text { I.7 }\end{array}$ & Cord. ZC. $375^{\circ}$ \\
\hline 8 & $944 \quad 41.85$ & -5355 & Cord. GC. I 3388 \\
\hline 9 & $937 \quad 56.93$ & $\begin{array}{lll}-52 & 4 & 8.4\end{array}$ & Cord. ZC. 2916 \\
\hline IO & $\begin{array}{lll}9 & 33 & 8.66\end{array}$ & $\begin{array}{lll}-48 & 53 & 36.8\end{array}$ & Cord. GC. I 3145 \\
\hline
\end{tabular}

The above observations were made under very unfavorable conditions. The object was barely visible in dark field, and it faded away under the faintest illumination, so that I cannot claim any great accuracy in the results.

Cordoba 1897 July $\mathrm{I}_{5}$.

Fohn M. Thome.

\section{Benennung und Numerirung von kleinen Planeten.}

(354) Eléonora, 1893 A, découverte 1893 Jan. 17. (416) Vaticana, 1896 CS, découverte 1896 Mai 4. A. Charlois. Planet $1896 \mathrm{DC}$, entdeckt am 28. Dec. 1896 von Charlois, hat die Nummer (425) erbalten. A. Berberich.

Pianeta (101) Helena. Correzione dell' effemeride (Veröff. R. I. Nr. 5): Agosto ${ }^{7}-2^{\mathrm{m}} \mathrm{I}^{5}-22^{\mathrm{s}} \mathbf{2}$. A. Antoniazzi. Pianeta (184) Dejopeja. Correzione dell' effemeride (Veröff. R. I. Nr. 5): Agosto 2 I - I I $^{\mathbf{3}}-0^{\prime} 7$. Gr. I 2. I. $A$. Abetti. Pianeta (186) Celnta. Correzione dell' effemeride (Veröff. R. I. Nr. 4): Agosto $18-46^{5}-7: 6$. A. Antoniazzi. Planet (187) Lamberta. Correction der Ephemeride (Veröf. R. I. Nr. 4): Aug. 18-6 - 1'ı. W. Villiger. Planet (194) Prokno. Correction der Ephemeride (Berl. Jahrb. 1899): Aug. $22+1^{\mathrm{m}} \mathrm{r}^{\mathrm{s}} 25+47^{\prime \prime} \mathrm{r}$. R. Luther. Planet (416) Vaticana. Correction der Ephemeride (A. N. 3438): Aug. 8 + 9.17 +63"8. W. Villiger. I75. - Correctionen von Planetenephemeriden. 175. 